\title{
Relation of pretreatment sequence diversity in NS5A region of HCV genotype 1 with immune response between pegylated- INF/ribavirin therapy outcomes
}

\author{
A. T. L. de Queiróz, ${ }^{1}$ V. Maracaja-Coutinho, ${ }^{2}$ A. C. G. Jardim, ${ }^{3}$ P. Rahal, ${ }^{4}$ I. M. V. G. de \\ Carvalho-Mello ${ }^{4}$ and S. R. Matioli ${ }^{1}{ }^{1}$ Department of Genetics and Evolutive Biology, Biosciences Institute, USP - São Paulo \\ University; ${ }^{2}$ Biochemistry Department, Chemistry Institute, USP - São Paulo University; ${ }^{3}$ Department of Biology, UNESP - Instituto de Biociências, \\ Letras e Ciências Exatas, São Paulo State University; and ${ }^{4}$ Viral Immunology Laboratory, Butantan Institute, São Paulo, Brasil
}

Received October 2009; accepted for publication February 2010

\begin{abstract}
SUMMARY. Hepatitis C virus (HCV) infection frequently persists despite substantial virus-specific immune responses and the combination of pegylated interferon (INF)- $\alpha$ and ribavirin therapy. Major histocompatibility complex class I restricted $\mathrm{CD}^{+} \mathrm{T}$ cells are responsible for the control of viraemia in $\mathrm{HCV}$ infection, and several studies suggest protection against viral infection associated with specific HLAs. The reason for low rates of sustained viral response (SVR) in HCV patients remains unknown. Escape mutations in response to cytotoxic $\mathrm{T}$ lymphocyte are widely described; however, its influence in the treatment outcome is ill understood. Here, we investigate the differences in CD8 epitopes frequencies from the Los Alamos database between groups of patients that showed distinct response to pegylated $\alpha$-INF with ribavirin therapy and test evidence of natural
\end{abstract}

selection on the virus in those who failed treatment, using five maximum likelihood evolutionary models from PAML package. The group of sustained virological responders showed three epitopes with frequencies higher than Nonresponders group, all had statistical support, and we observed evidence of selection pressure in the last group. No escape mutation was observed. Interestingly, the epitope VLSDFK TWL was 100\% conserved in SVR group. These results suggest that the response to treatment can be explained by the increase in immune pressure, induced by interferon therapy, and the presence of those epitopes may represent an important factor in determining the outcome of therapy.

Keywords: genotype 1, hepatitis C virus, hepatitis, immune response, NS5A.

\section{INTRODUCTION}

Hepatitis C Virus (HCV), a positive-strand RNA virus of the Flaviviridae family, is a major cause of chronic liver disease. An estimated 130 million individuals are infected worldwide [1], and about $70 \%$ of infections become chronic, linked with high rate of mutations in the HCV genome that leads to the

\footnotetext{
Abbreviations: BEB, Bayes empirical Bayes; dN, nonsynonymous substitution rate; $\mathrm{dN} / \mathrm{dS}$, nonsynonymous/synonymous substitution rate ratio; $\mathrm{dS}$, synonymous substitution rate; ETR, end of treatment response; $\mathrm{HCV}$, hepatitis $\mathrm{C}$ virus; $\mathrm{HIV}$, human immunodeficiency virus; INF, interferon; ISDR, interferon sensitivity-determining region; LRT, likelihood ratio test; MHC, major histocompatibility complex; NR, non-responders; PKR, protein kinase R; RT-PCR, reverse transcriptase-polimerase chain reaction; SVR, sustained virological response.

Correspondence: Isabel M. V. G. de Carvalho-Mello, Laboratório de Imunologia Viral, Instituto Butantan, Av Vital Brasil n ${ }^{\circ} 1500$, CEP 05503-900, Butantã, São Paulo, SP, Brasil. E-mail: imvgcmello@ butantan.gov.br
}

occurrence and selection of escape variants, which contribute with persistence of infection and chronic cases that are often associated with cirrhosis and hepatocellular carcinoma a [2-4].

The NS5A is a nonstructural HCV protein associated with several functions such as transcriptional repressor and associated via the interferon (INF)-sensitive determining region (ISDR), with influence on INF activity.[5]. The ISDR inhibits the cellular RNA-activate protein kinase R (PKR), which is an effector of INF antiviral activity. The NS5A might function as viral transactivator [6]. The NS5A is the major nonstructural protein associated with INF resistance; however, no specific explanation about which variables that influence the therapy outcome or how is the influence of immune pressure on this region was well described.

Furthermore, only about $30-40 \%$ of the patients treated with the current therapy with $\alpha$ - INF present sustained virological response (SVR), and the reasons why therapy is effective in only about half of cases are unknown [7]. The cytokine is involved in innate immune responses against 
viral infections and Th1 polarization [8,9]. In addition, it was reported that co-administration of INF- $\alpha$ with HIV-1 DNA vaccine increases cellular and humoral immune response [10].

There is growing evidence that INF- $\alpha$ therapy is associated with the increase in HCV-specific T-cell immunity in chronic hepatitis $C[11,12]$, nevertheless, the influence of immune system on the outcome of infection has not been understood.

Several studies suggest an important role of the adaptive T-cell response for control of acute hepatitis C virus (HCV) infection [13], and the association between the expression of particular major histocompatibility complex (MHC) class I alleles and the natural course of HCV infection has been described, suggesting an important role of virus infection control [14]. Moreover, an strong $\mathrm{CD}^{+}$T-cell immunity in acute resolving hepatitis $\mathrm{C}$ is matched by strong and sustained $\mathrm{CD}^{+}$T-cell proliferation to multiple recombinant structural and nonstructural viral proteins [15].This virusspecific $\mathrm{CD}^{+}$T-cell response in acute resolving HCV infection is also vigorous and multispecific, targeting up to eight to twelve epitopes $[16,17]$.

In studies on HCV infection, it was found strong association between viral persistence and escape mutations [18]. However, the determinants of viral escape, as well as its relative contribution to T-cell failure and $\mathrm{HCV}$ persistence, remain not well characterized.

The aim of this study is to evaluate the influence of immune response on entire NS5A region of viral quasispecies before the INF therapy in pretreatment patients infected with genotype 1 virus and its consequences on viral protein evolution regarding the presence of mapped epitopes.

\section{MATERIALS AND METHODS}

\section{Patients}

For this study, eleven patients infected chronically with HCV RNA genotypes $1 \mathrm{a}$ or $1 \mathrm{~b}$ from the Hepatology Department of the São José do Rio Preto University Hospital were included. All patient characteristics, therapy protocols and Ethics Committee were described by Jardim et al. [19]. In this analysis, the patients were classified into two groups, according to their response to therapy: three patients (2-4) were classified as SVR. Eight patients (5-12) were nonresponders (NR) to the therapy. SVR was defined as absence of HCV RNA in plasma by qualitative PCR 6 months after the end of the therapy. NR was defined as continued presence of HCV RNA in plasma during treatment and 6 months after and HCV RNA-negative at the end of the therapy but experienced a rebound in HCV viraemia afterwards. The patient 1 was excluded from analysis because the NS5A region was not fully sequenced. The SVR and NR groups have similar age means (39.6 and 39.7, respectively); however, most of the patients were men (one woman and two men in SVR group and one woman and seven men in
NR group). The SVR group has two patients infected with 1a genotype virus and one with $1 \mathrm{~b}$. The NR group has four $1 \mathrm{a}$ virus genotype-infected patients and four infected with $1 \mathrm{~b}$ genotype virus. The Nucleotide sequence data reported in this article are available in GenBank under the accession numbers EU309511 to EU309673.

\section{Cloning and sequencing HCV genome}

For each patient, 15 clones of RT-PCR-amplified fragment of the virus were sequenced, except for patients 3 and 5, who have lower numbers of sequenced clones (14 clones). The sequence length was 1344 nucleotides, representing all NS5A regions. Thus, 163 full-length NS5A sequences were analysed in this work. All protocols of RNA extraction, RT-PCR, NS5A amplification, cloning and sequencing the HCV RNA from patients are described elsewhere [19].

\section{Sequence analysis}

Sequences were aligned with Muscle, [20] and the alignments were visually edited using GeneDoc [21]. The epitope mapping was performed using the $\mathrm{CD} 8^{+}$epitopes from $\mathrm{HCV}$ Immunology database [22]. For this, all the 21 NS5A epitopes were used to evaluate the frequency of each epitope between two groups. To determine potential positive selection, we used two approaches. A maximum likelihood approach (ML) using codeml program of PAML4 package [23] was employed for the entire NS5A region. For each epitope with statistical difference between both groups, we estimate the $\mathrm{dN} / \mathrm{dS}$ ratio, $\mathrm{dS}$ and $\mathrm{dN}$ using the yn00 program of PAML4 package.

\section{The $d N / d S, d N$ and $d S$ estimation}

For $\mathrm{dN} / \mathrm{dS}$ ratio, $\mathrm{dN}$ and $\mathrm{dS}$ epitope analysis, all values were measured using the method of Yang and Nielsen [24]. For each epitope, the nucleotide sequence alignments were performed and used for the analysis. To calculate the $\mathrm{dN} / \mathrm{dS}$ values for all epitope quasispecies between two groups, all pairwise comparisons for an epitope were performed, and values were averaged overall.

\section{Maximum likelihood analysis}

Maximum likelihood topologies were estimated using PhyML v2.44 [25] and used in ML analysis implemented in codeml. The site models were used to detect positive selection without averaging the $\mathrm{dN} / \mathrm{dS}$ ratio throughout the phylogenetic tree. The M0 (one ratio), M1a (neutral), M2a (selection), M7 (beta) and M8 (beta\&W) codon-based ML substitution models were used to measure the $\mathrm{dN} / \mathrm{dS}$ ratio and to test the evidence of positive selection by comparing the likelihood values of the different nested models using the. The Bayes Empirical Bayes approach was performed if the LRT suggests 
evidence of positive selection to determine which individual codon is subjected to positive selection.

\section{Statistic analysis}

Statistical difference in the parameters was determined between different patients' groups by Mann-Whitney nonparametric test for numerical variables. The comparisons of presence/absence of epitopes among the patient groups were made using the G statistics. We also applied the conservative Bonferroni's correction for multiple comparisons.

The LRT statistics $(2 \Delta \ell)$ was performed and compared against $\chi^{2}$, with critical values to be 5.99 and 9.21 at $5 \%$ and $1 \%$ significance levels. The first pair M1a (neutral) vs M2a (selection) and M7 (beta) vs M8 (beta\&W) were used for verify evidence of positive selection as suggested by Yang [23].

\section{RESULTS}

\section{Mapping of $\mathrm{CD}^{+} \mathrm{HCV}$-specific epitopes in both infected patient groups in NS5A protein}

First, we performed a screening of epitopes from $\mathrm{HCV}$ Immunology Database in NS5A translated protein sequence from SVR group $(N=44)$, as well as for protein sequence from the NR group $(N=119)$. Thus, 21 epitopes overall were used for the mapping, representing all from NS5A region, and these results are presented in Table 1.

Three of them have showed no matches in NS5A protein in both SVR and NR groups (epitopes: VLDSFKTWL, LPKLPGVPF and QLSAPSLKATCTANH), and one has no match in SVR group and low frequency in NR group $(5.04 \%$ of the epitope KFPPALPIW). Five epitopes have demonstrated higher frequency rates in the NR group when compared with SVR group, and eight showed higher rates in SVR group, but without statistical significance.

Overall, the frequency of HCV-specific epitopes was lower in NR group, when compared with SVR group, but this difference was not statistically significant. Four epitopes showed statistical differences with the $G$ test between patient response groups. Three of them have shown higher frequency rate in SVR group (epitopes: VLSDFKTWL, ARGSPPSVASSSASQLSAPS and SVASSSASQLSA). However, the epitope VLSDFKTWL showed 100\% frequency compared to $48 \%$ in NR group. Only the epitope LTDPSHITA had higher frequency rate in NR group, with 98\% compared to $65 \%$ in SVR group. All epitopes that showed statistical significance were identified as HLA allele A-binding epitopes (alleles: A1, A2 and both).

\section{Analysis of selection pressure in patient groups}

Maximum likelihood methods were used to assess amino acid variation and identify targets of positive selection, and
Table 1 Frequencies of epitopes in the HCV quasispecies NS5a protein sequences from the patient groups (Genotype 1 $\mathrm{a} / \mathrm{b})$

\begin{tabular}{llcc}
\hline EPITOPE & Allele & SVR & NR \\
\hline VLSDFKTWL & A2 & 100 & $48.73^{*}$ \\
VLDSFKTWL & A2 & 0 & 0 \\
SDFKTWLKA & Patr-B*1601 & 65.90 & 47.89 \\
DFKTWLQSKL & NA & 34.09 & 50.42 \\
LPKLPGVPF & B35 & 0 & 0 \\
MWSGTFPINAY & Patr-A*0601 & 63.63 & 49.57 \\
LLREEVSFRV & A2 & 34.09 & 49.57 \\
HEYPVGSQL & B60, H-2k & 63.63 & 48.73 \\
PCEPEPDVAVL & B35 & 31.81 & 47.05 \\
EPEPDVAVL & B35 & 31.81 & 47.05 \\
LTDPSHITA & A1 & 65.90 & $98.31^{*}$ \\
ARGSPPSVASSSASQLSAPS & A1/A2 & 65.90 & $25.21^{*}$ \\
SVASSSASQLSA & A1/A2 & 65.90 & $25.21^{*}$ \\
QLSAPSLKATCTANH & NA & 0 & 0 \\
NHDSPDAEL & B38 & 61.36 & 49.57 \\
SPDAELIEANL & A2 & 65.90 & 49.57 \\
ELIEANLLW & A25 & 65.90 & 49.57 \\
ILDSFDPLV & A2 & 31.81 & 10.92 \\
LDSFDPLVA & Patr-B*0401 & 65.90 & 47.89 \\
REISVPAEIL & B60 & 31.81 & 49.57 \\
KFPPALPIW & A*2402 & 0 & 5.04 \\
\hline
\end{tabular}

NA, not available; SVR, sustained virological response group; NR, non-responders group.

*Represents different frequencies between patient groups with $P$-value $<0.05$ (corrected with the Bonferroni's procedure); All frequencies are expressed in percentage.

Table 2 Results of selective pressure between the patient groups

\begin{tabular}{lllll}
\hline $\begin{array}{l}\text { Treatment } \\
\text { response }\end{array}$ & $\begin{array}{l}\text { dN/dS } \\
\text { ratio }\end{array}$ & $\begin{array}{l}\text { M2a } \\
\text { vs M1a }\end{array}$ & $\begin{array}{l}\text { M8 } \\
\text { vs M7 }\end{array}$ & $\begin{array}{l}\text { Positive selected } \\
\text { AA (position) }\end{array}$ \\
\hline SVR & 0.09 & 0.367 & 4.058 & ND \\
NR & 0.09 & 0.023 & $13.376^{*}$ & L(2130), P(2319) \\
Both groups & 0.1 & 0.001 & $15.49^{*}$ & L(2130), P(2319) \\
\hline
\end{tabular}

ND, not detected; dN/dS, nonsynonymous/synonymous substitution rate ratio; AA, aminoacid; SVR, sustained virological response group; NR, Non-responders group. *Represents statistically significant $P$-value $<0.01$, in likelihood ratio test.

all values are presented in Table 2 . The mean $\mathrm{dN} / \mathrm{dS}$ value of NS5A region from SVR, NR and both groups was measured with MO and showed no statistical support. The comparison of first pair M1a (neutral) and M2a (selection) showed no statistical support in NR and SVR groups; however, the second pair M7 (beta) and M8 (beta\&w) analysed with the 
LRT statistic suggests that M8 model was selected $(P<0.01)$, providing evidence of varying selection pressure at individual sites across the NS5A region of NR group and in both groups. As LRT suggested that these groups are under positive selection, the Bayes empirical Bayes (BEB) method was used to calculate the posterior probabilities that each codon is from the site class of positive selection under model M8, thus, indicating that the aminoacid L in the position 2130, according to H77 reference strain, and P in the position 2319 as being positively selected, with a posterior probability $>95 \%$.

\section{$d N / d S$ ratio, $d S$ and $d N$ measure of significant epitopes}

For the four epitopes that showed statistical differences between two groups, a measure pairwise analysis of $\mathrm{dN} / \mathrm{dS}$ ratio, the proportion of synonymous and the proportion of nonsynonymous mutations of epitope region were calculated. The results are shown in Table 3. Three of them have higher $\mathrm{dN} / \mathrm{dS}$ ratio in NR group, and one of them showed higher ratio in SVR group, all of them with statistical

Table 3 Values of $\mathrm{dN} / \mathrm{dS}$, $\mathrm{dN}$ and $\mathrm{dS}$ for each epitope from group SVR and NR

\begin{tabular}{lllll}
\hline Epitope & Group & $\mathrm{dN} / \mathrm{dS}$ & $\mathrm{dN}$ & $\mathrm{dS}$ \\
\hline VLSDFKTWL & SVR & 0.03 & 0.02 & 0.76 \\
& NR & $0.04^{*}$ & $0.03^{*}$ & $0.91^{*}$ \\
LTDPSHITA & SVR & $0.018^{*}$ & $0.026^{*}$ & 0.79 \\
& NR & 0.001 & 0.001 & $0.86^{*}$ \\
ARGSPPSVASSSASQLSAPS & SVR & 0.02 & 0.01 & 0.29 \\
& NR & $0.09^{*}$ & 0.01 & $0.34^{*}$ \\
SVASSSASQLSA & SVR & 0.06 & 0.02 & 0.25 \\
& NR & $0.12^{*}$ & $0.03^{*}$ & 0.25 \\
\hline
\end{tabular}

$\mathrm{dN} / \mathrm{dS}$, nonsynonymous/synonymous substitution rate ratio; $\mathrm{dN}$, nonsynonymous substitution rate; DS, synonymous substitution rate; SVR, sustained virological response group; NR, non-responders group.

*Represents statistically significant $P$-value $<0.05$, in Mann-Whitney Test comparing SVR and NR groups. support. Interestingly, the epitopes showed higher ratios in groups where the frequency of them was lower. In $\mathrm{dN}$ measure, one epitope showed no difference between groups. Two of them showed $\mathrm{dN}$ value higher in NR group, and one had $\mathrm{dN}$ value higher in SVR group, with statistical support. The dS measure provides higher values in NR group of three epitopes with statistical support. One epitope had no difference in dS values in both groups.

\section{Aminoacid change in epitopes with statistical difference}

As shown in Table 4, no viral sequence variation was detected within epitope VLSDFKTWL in SVR group. However, in NR group, it was observed two aminoacid changes in position 3 of epitope (P3) serine was substituted by threonine with $37.8 \%$ and substituted by alanine with $12.6 \%$. Sequence analysis of epitope LTDPSHITA has demonstrated aminoacid change in second position (P2), a threonine was substituted by serine in $34 \%$ of sequences of the SVR group. The aminoacid changes in NR group were $<2 \%$. The epitope ARGSPPSVASSSASQLSAPS showed aminoacid change in position 8 , where a valine was substituted by leucine with $33.9 \%$ in SVR group. Another aminoacid changes occurred in this epitope with $2.2 \%$ of frequency. Arginine was substituted by glycine at second position, and serine was substituted by leucine in position 12 . In NR group, this epitope presented aminoacid change in position 8 , where valine was substituted by leucine with $63 \%$ of frequency and valine to methionine with $12.6 \%$. The epitope SVASSSASQLSA showed aminoacid variation in position 2 , where valine was substituted by leucine with $33.9 \%$ of frequency in SVR group and $63 \%$ in NR group. Valine was substituted by methionine in the same position with $12.6 \%$ frequency in NR group. None of the aminoacids changes within epitopes had BEB analysis posterior probability evidence of positive selection.

\section{DISCUSSION}

Our study was performed to address the influence of $\mathrm{CD} 8^{+} \mathrm{T}$ cell-mediated inhibition of HCV on the outcome of peg-INF therapy. We identified four epitopes previously described in

Table 4 Mutations observed within epitopes with statistically significant difference between treatment response groups

\begin{tabular}{|c|c|c|c|c|}
\hline & VLSDFKTWL & LTDPSHITA & ARGSPPSVASSSASQLSAPS & SVASSSASQLSA \\
\hline SVR & $\ldots \ldots \ldots$ &.$S \ldots \ldots$ & $\begin{array}{l}\ldots \ldots L\llcorner\ldots \ldots \\
\ldots \ldots \ldots L \ldots L \ldots \ldots \\
. G \ldots \ldots L \ldots \ldots \ldots \ldots\end{array}$ & $\begin{array}{l}. \mathrm{L} \ldots \ldots \\
. \mathrm{L} \ldots \mathrm{L} \ldots\end{array}$ \\
\hline NR & $\begin{array}{l}\ldots \mathrm{T} \ldots \ldots \\
\ldots \mathrm{A} \ldots \ldots\end{array}$ & $\begin{array}{l}\ldots \mathrm{S} \ldots \\
\ldots \mathrm{T} \ldots\end{array}$ & $\begin{array}{l}\ldots \ldots \text { м. } \ldots \ldots \ldots \\
\ldots \ldots\llcorner. \ldots \ldots \ldots\end{array}$ & $\begin{array}{l}. \mathrm{M} \ldots \ldots \ldots \\
. \mathrm{L} \ldots \ldots \ldots\end{array}$ \\
\hline
\end{tabular}

SVR, sustained virological response group; NR, non-responders group. 
NS5A region [22] with significant differences between the patient groups, despite the small number of patient in our analysis. The epitopes VLSDFKTWL, ARGSPPSVASSS ASQLSAPS and SVASSSASQLSA showed higher frequencies in sustained viral response (SVR) group when compared to the NR group. Thus, this data might indicate that those epitopes, specially the VLSDFKTWL, induced by INF, promote immune response against HCV and may drive the treatment outcome. Despite the epitope LTDPSHITA, which had shown higher aminoacid variation in SVR compared to NR group, it could be explained as an ineffective response against this epitope in NR group, therefore, this epitope could not have effect in the clearance of HCV.

It is important to note that epitope VLSDFKTWL had $100 \%$ of matches in protein sequences of SVR group and $48 \%$ in NR group. However, the NR group has two distinct treatment failure patients: complete non-response patients, defined as continued presence of HCV RNA in plasma during treatment and 6 months after the end of the treatment. Patients who were HCV RNA-negative at the end of the therapy but experienced a rebound in HCV viraemia afterwards were classified as end of treatment response (ETR). About $75 \%$ of patients (three of them) with sequence variation presented complete nonresponse to the treatment, and just 25\% (one patient) showed ETR. This result suggests that the presence of this epitope plays an important role in specific $\mathrm{CD}^{+}$response against $\mathrm{HCV}$ control, and mutations in this region could affect in the treatment outcome. However, because of our small number of patients, future studies are needed to provide more information about the course of HCV infection and t-cell epitope dynamics during therapy.

We investigated the evidence of positive selection in HCV NS5A sequences of patients using evolutionary models as described in the Methods section. We observed the same value of $\mathrm{dN} / \mathrm{dS}$ ratio in both groups $(\mathrm{dN} / \mathrm{dS}$ ratio $=0.09)$ and a similar value when we analysed them together. All of the three values showed evidence of purifying selection. However, the straightforward use of this ratio is rarely effective to detect positive selection, because the ratio over all sites is often $<1$ [23]. In the ML analysis of selection models, the results showed no evidence of positive selection in the SVR group, which suggests that immune response and the peg-INF therapy were effective to clear the HCV before the emergence of the escape mutations which improve the viral fitness, leading to the treatment failure. Nevertheless, in the NR group and in both groups together, we observed evidence of positive selection. This finding suggests that the failure of therapy is associated with the NS5A region variation, despite none of the two amino acids were shown to be under positive selection by the Bayesian analysis in the epitopes regions. The evidence of positive selection in regions out of epitopes indicates that the mutations in NS5A protein keep the viral replication in the presence of the drugs and compensate the viral fitness.
Several studies have reported that sequence variation in NS5A region is associated with IFN responsiveness, specifically the IFN sensitivity-determining region, RNA-activated protein kinase (PKR)-binding domain and V3 region [26-29]. Nevertheless, the role of CD8+ T cells in the control of $\mathrm{HCV}$ replication during INF therapy has been not well described. Our results show that the presence of epitope VLSDFKTWL is strongly associated with virus clearance during treatment, suggesting that the CD8+ T cells' specificity to the HCV could increase and promote a strong immune response, being an important factor in the treatment success. Interestingly, this epitope was described in cytoplasmatic retention signal region [30], because this region was not been associated with immune response activity or therapy effectiveness.

In agreement with several other studies, we found evidence of an important role of specific CD8+ T-cells epitopes in control of viraemia and association with viral persistent in chronically evolving patients and escape mutations, even with the small sample size [31-33].

Because escape mutations could impair HLA binding or loss of processing during MHC presenting process, we then assessed the specific mutations in epitopes regions to verify which positions have sequence variation and compare to information about HLA epitopes in the SYFPEITHI database [34]. With the exception of the epitope SVASSSASQLSA, which showed the mutation valine to leucine at second position of the epitopes, all other mutations were not associated with expected aminoacids in anchor positions or preferred residues. In this epitope, a valine mutates to leucine, which is an expected anchor aminoacid to this position in allele HLA A1. The epitope VLSDFKTWL showed no sequence variation in SVR group; however, in the NR group, the position 3 serine was substituted by threonine with $37.8 \%$ and by alanine with $12.6 \%$. These results reinforce the association between the role of $\mathrm{CD}^{+}{ }^{+}$-specific immune response against $\mathrm{HCV}$ and the therapy, because those aminoacids are not expected as preferred residues as described in SYFPEITHI database from allele A2, indicating that are escape mutations. T-cell escape mutations resulting in complete abrogation of HLA binding or disruption of processing will not be presented on the surface of the cell and are thus invisible to immune system. It is important to note that, in addition to the hypothesis suggested previously, host factors, such as the genetic restriction of the immune response, T-cell receptor diversity or strong $\mathrm{CD} 4^{+} \mathrm{T}$-cell help [35-37], may also contribute to the observed viral escape in NR group.

We decide to focus on the epitope regions and evaluate the $\mathrm{dN} / \mathrm{dS}$ ratio, $\mathrm{dS}$ and $\mathrm{dN}$ for each epitope in both groups to verify statistical differences. For three epitopes that showed higher frequencies in SVR group, we found $\mathrm{dN} / \mathrm{dS}$ ratio lower than NR, suggesting that immune system perform selective pressure on this epitopes. Despite the epitope found LTDPSHITA showed dN/dS ratio higher in SVR group, 
it could be explained by the higher $\mathrm{dS}$ value in NR group, suggesting unspecific immune response in patients from NR group, because this group showed no response to treatment.

In summary, mapping 21 NS5A epitopes in our eleven HCV chronically evolving patients under peg-INF therapy, we could show evidence of at least one T-cell HCV-specific epitope with association on the treatment outcome. In addition, evolutionary models were used to confirm the presence of immune pressure on the virus. Taken together, these results have important implications to understand the $\mathrm{HCV} /$ immune system dynamics during the treatment period, indicating that immune system plays an important role in $\mathrm{HCV}$ clearance during treatment period.

\section{FINANCIAL DISCLOSURE}

This work was financially supported by CAPES.

\section{REFERENCES}

1 Lauer GM, Walker BD. Hepatitis C virus infection. $N$ Engl J Med 2001; 345(1): 41-52.

2 Farci P, Shimoda A, Coiana A et al. The outcome of acute hepatitis $\mathrm{C}$ predicted by the evolution of the viral quasispecies. Science 2000; 288(5464): 339-344.

3 Lindenbach BD, Rice CM. Unravelling hepatitis C virus replication from genome to function. Nature 2005; 436: 933-938.

4 Hoofnagle JH. Course and outcome of hepatitis C. Hepatology 2002; 36(5): 21-29.

5 Ghosh AK, Steel R, Meyer K, Ray R, Ray RB. Hepatitis C virus NS5A protein modulates cell cycle regulatory genes and promotes cell growth. J Gen Virol 1999; 80(5): 1179 1183.

6 Gale MJ, Korth MJ, Tahg NM et al. Evidence that Hepatitis C virus resistence to interferon is mediated through repression of PKR protein kinase by the nonstructural $5 \mathrm{~A}$ protein. Virology 1997; 230(2): 217-227.

7 Nunez M, Soriano V. Current concepts in the management and treatment of hepatitis $\mathrm{C}$ in HIV-infected patients. Ann Hepatol 2005; 4(3): 151-160.

8 Neurath MF, Finotto S, Glimcher LH. The role of Th1/Th2 polarization in mucosal immunity. Nat Med 2002; 8(6): $567-573$.

9 Tough DF. Type I interferon as a link between innate and adaptive immunity through dendritic cell simulation. Leuk Lymphoma 2004; 45(2): 257-264.

10 Jiang W, Ren L, Jin N. HIV-1 DNA Vaccine efficacy is enhanced by coadministration with plasmid encoding INFalpha. J Virol Methods 2007; 146: 266-273.

11 Tang KH, Herrmann E, Cooksley $\mathrm{H}$ et al. Relationship between early HCV kinetics and T-cell reactivity in chronic hepatitis $\mathrm{C}$ genotype 1 during peginterferon and ribavirin therapy. J Hepatol 2005; 43(5): 776-782.

12 Pugnale P, Herrmann E, Neumann AU et al. Hepatitis C viral kinetics in plasma and peripheral blood mononuclear cells during pegylated interferon-alpha2a/ribavirin therapy. J Hepatol 2008; 48(6): 932-938.
13 Dustin LB, Rice CM. Flying under the radar: the immunobiology of hepatitis C. Апnu Rev Immunol 2007; 25: 71-99.

14 McKiernan SM, Hagan R, Curry M et al. Distinct MHC class I and II alleles are associated with hepatitis C viral clearance, originating from a single source. Hepatology 2004; 40(1): 108-114.

15 Gerlach JT, Diepolder HM, Jung MC et al. Recurrent of hepatitis $\mathrm{C}$ virus after loss of virus specific $\mathrm{CD} 4^{+}$T-cell response in acute hepatitis C. Gastroenterology 1999; 117(4): 933-941.

16 Gruner NH, Gerlach TJ, Jung MC et al. Association of hepatitis $\mathrm{C}$ virus-specific CD8+ T cells with viral clereance in acute hepatitis C. J Infect Dis 2000; 181(5): 1528-1536.

17 Lechhner F, Wong DK, Dunbar PR et al. Analysis of sucessful immune responses in persons infected with hepatitis C virus. J Exp Med 2000; 191(9): 1499-1512.

18 Cox AL, Mosbruger T, Mao Q et al. Cellular immune selection with hepatitis $\mathrm{C}$ virus persistence in humans. J Exp Med 2005; 201(11): 1741-1752.

19 Jardim AC, Yamasaki LH, Queiroz ATL et al. Quasispecies of hepatitis $\mathrm{C}$ virus genotype 1 and treatment outcome with Peginterferon and Ribavirin. Infect Genet Evol 2009; 9(4): 689-698.

20 Edgar RC. MUSCLE: multiple sequence alignment with high accuracy and high throughput. Nucleic Acids Res 2004; 35(5): 1792-1797.

21 Nicholas KB, Nicholas HBJ, Deerfield DW. GeneDoc: analysis and visualization of genetic variation. Embnew News 1997; 4: 1-4.

22 Yusim K, Richardson R, Tao N et al. Los alamos hepatitis C immunology database. Appl Bioinformatics 2005; 4(4): 217 225.

23 Yang Z. PAML 4: phylogenetic analysis by Maximum Likelihood. Mol Biol Evol 2007; 24(8): 1586-1591.

24 Yang Z, Nielsen R. Estimating synonymous and nonsynonymous substitution rates under realistic evolutionary models. Mol Biol Evol 2000; 17(1): 32-43.

25 Guindon S, Delsuc F, Dufayard JF, Gascuel O. Estimating Maximum Likelihood Phylogenies with PhyML. Methods Mol Biol 2009; 537: 113-137.

26 Enomoto N, Sakuma I, Asahina Y et al. Mutations in the nonstructural protein $5 \mathrm{~A}$ gene and response to interferon in patients with chronic hepatitis $\mathrm{C}$ virus $1 \mathrm{~b}$ infection. $N$ Engl J Med 1996; 334(2): 77-81.

27 Gale MJ, Korth MJ, Katze MG. Repression of the PKR protein kinase by the hepatitis C virus NS5A protein: a potential mechanism of interferon resistance. Clin Diagn Virol 1998; 3: 157-162.

28 Nousbaum J, Polyak SJ, Ray SC et al. Prospective characterization of full-length hepatitis C virus NS5A quasispecies during induction and combination antiviral therapy. J Virol 2000; 74(19): 9028-9038.

29 Duverlie G, Khorsi H, Castelain S et al. Sequence analysis of the NS5A protein of European hepatitis C virus 1b isolates and relation to interferon sensitivity. J Gen Virol 1998; 79(6): 1373-1381.

30 Ubani S, Uggeri J, Matsuura Y et al. Identification of immunodominant hepatitis $\mathrm{C}$ virus (HCV)-specific cytotoxic 
T-cell epitopes by stimulation with endogenously synthesized HCV antigens. Hepatology 2001; 33(6): 1533-1543.

31 Guglietta S, Garbuglia AR, Pacciani V et al. Positive selection of cytotoxic T lymphocyte escape variants during acute hepatitis C virus infection. Eur J Immunol 2005; 35(9): 2627-2637.

32 Tester I, Smyk-Pearson S, Wang P et al. Immune evasion versus recovery after acute hepatitis C virus infection from a shared source. J Exp Med 2005; 201(11): 17251731.

33 Timm J, Lauer GM, Kavanagh DG et al. CD8 epitope escape and reversion in acute HCV infection. J Exp Med 2004; 200(12): 1593-1604.
34 Rammensee H, Bachmann J, Emmerich NP, Bachor OA, Stevanović S. SYFPEITHI: database for MHC ligands and peptide motifs. Immunogenetics 1999; 4: 213-219.

35 Neumann-Haefelin C, McKiernan S, Ward S et al. Dominant influence of an HLAB27 restricted CD8+ T cell response in mediating HCV clearance and evolution. Hepatology 2006; 43(3): 563-572.

36 Meyer-Olson D, Shoukry NH, Brady KW et al. Limited T cell receptor diversity of HCV-specific T cell responses is associated with CTL escape. J Exp Med 2004; 200(3): 307-319.

37 Grakoui A, Shoukry NH, Woollard DJ et al. HCV persistence and immune evasion in the absence of memory $\mathrm{T}$ cell help. Science 2003; 302(5645): 659-662. 